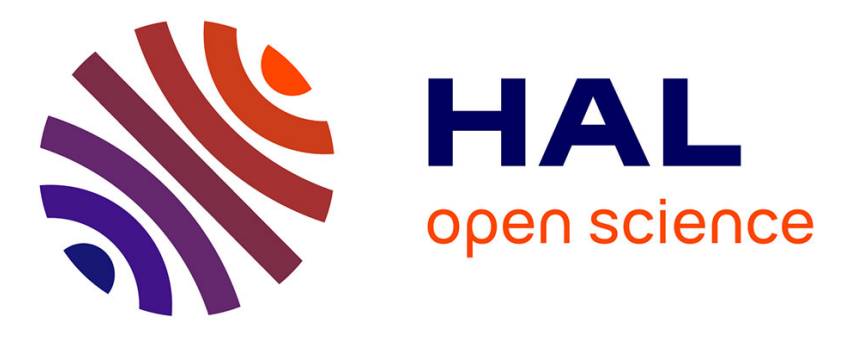

\title{
Adaptation in serious games for upper-limb rehabilitation: An approach to improve training outcomes
}

Nadia Hocine, Abdelkader Gouaich, Stefano A. Cerri, Denis Mottet, Jérome Froger, Isabelle Laffont

\section{To cite this version:}

Nadia Hocine, Abdelkader Gouaich, Stefano A. Cerri, Denis Mottet, Jérome Froger, et al.. Adaptation in serious games for upper-limb rehabilitation: An approach to improve training outcomes. User Modeling and User-Adapted Interaction, 2015, 25 (1), pp.65-98. 10.1007/s11257-015-9154-6 . lirmm01121345

\section{HAL Id: lirmm-01121345 \\ https://hal-lirmm.ccsd.cnrs.fr/lirmm-01121345}

Submitted on 28 Feb 2015

HAL is a multi-disciplinary open access archive for the deposit and dissemination of scientific research documents, whether they are published or not. The documents may come from teaching and research institutions in France or abroad, or from public or private research centers.
L'archive ouverte pluridisciplinaire HAL, est destinée au dépôt et à la diffusion de documents scientifiques de niveau recherche, publiés ou non, émanant des établissements d'enseignement et de recherche français ou étrangers, des laboratoires publics ou privés. 


\title{
Adaptation in serious games for upper-limb rehabilitation:
}

\section{An approach to improve training outcomes}

\author{
Nadia Hocine - Abdelkader Gouaïch • \\ Stefano A. Cerri - Denis Mottet . \\ Jérome Froger · Isabelle Laffont
}

Received: date / Accepted: date

\begin{abstract}
In this paper, we propose a game adaptation technique that seeks to improve the training outcomes of stroke patients during a therapeutic session. This technique involves the generation of customized game levels, which difficulty is dynamically adjusted to the patients' abilities and performance. Our goal was to evaluate the effect of this adaptation strategy on the training outcomes of post-stroke patients during a therapeutic session. We hypothesized that a dynamic difficulty adaptation strategy would have a more positive effect on the training outcomes of patients than two control strategies, incremental difficulty adaptation and random difficulty adaptation. To test these strategies, we developed three versions of PRehab, a serious game for upper-limb rehabilitation. Seven stroke patients and three therapists participated in the experiment, and played all three versions of the game on a graphics tablet. The results of the experiment show that our dynamic adaptation technique increases movement amplitude during a therapeutic session. This finding may serve as a basis to improve patient recovery.
\end{abstract}

Keywords Adaptation - Serious games · Physical rehabilitation · Stroke · Upper-limb rehabilitation

"This is a draft version. Please find the final version of the paper in Springer http://link.springer.com/article/10.1007/s11257-015-9154-6"

\section{Introduction}

Stroke is a major cause of adult disability and the second leading cause of death worldwide (WHO, 2013; Rodrigo et al. , 2013). It is a medical emergency that occurs when the blood supply to the brain is disrupted. It can have serious consequences, such as sensorimotor deficiencies of the upper and

Address(es) of author(s) should be given 
lower contralateral limb, or cognitive impairments like hemineglect and aphasia (inability to understand or formulate speech). The abilities affected by stroke depend on the location, type and size of the lesion. Each patient is characterized by a specific combination of motor and cognitive deficits.

Stroke rehabilitation programs seek to help patients regain their functions and skills. In upper limb rehabilitation, these programs rely on strategies that are often task-oriented and based on reaching and grasping activities. Their objective is to improve training outcomes (e.g., motion control, movement amplitude, accuracy and eye-hand coordination). However, with repetitive activities, patients often become frustrated and tired. Therefore, therapists have to adapt the training parameters to patients while providing them with positive feedback. Many studies on motor learning have shown that the rehabilitation outcomes depend on the quality and the amount of physical activities (Kwakkel et al. , 1997) as well as on the patients' active participation in, and commitment to, the therapeutic session (Dobkin et al., 2005; Cirstea et al., 2007; Levin et al., 2010).

Serious games are promising support tools for individuals undergoing therapy. These video games are not intended for entertainment only. Nowadays, they are used in various application domains such as public policy, education and healthcare. From this perspective, the adaptation of a game to its application context may play a crucial role in ensuring the game's acceptability. Serious games for upper-limb rehabilitation (or rehabilitation games) seek to provide stroke patients with a motivating rehabilitation environment, by adapting the training intensity and challenges to their abilities and training needs (Pugnetti et al., 1998; Burke et al., 2009). These games also help therapists support patient motivation and gather quantitative measures on their progression.

A key feature of a successful game is its ability to provide the player with an adequate level of challenge. Most current difficulty adaptation techniques in entertainment games are based on the maximization of the player's motivation (Spronck et al., 2004; Natkin et al., 2007; Yannakakis et al., 2008). Those techniques use theories of motivation and flow (Czikszentmihalyi , 1991), but often fail to integrate the physical effort of actions into decisions. In the context of physical rehabilitation, the difficulty of the game is a key training factor, as it may influence patient performance. Most difficulty adaptation techniques in upper-limb rehabilitation games are inspired by motor learning theories and focus on the maximization of effort during the rehabilitation session. However, in the literature, rehabilitation games often rely on adhoc solutions to adapt the game. They use difficulty adaptation strategies which depend on game characteristics and generally lack reusability. Furthermore, although most studies on rehabilitation games have attempted to show their usability (Rabin et al. , 2011) and acceptability (Annett et al., 2009; Pirovano et al., 2012), they have failed to consider the effect of the difficulty strategy on patient performance.

In this paper, we first describe a generic difficulty adaptation technique for upper-limb rehabilitation games. The technique is based on the adapta- 
tion of pointing tasks and the generation of game levels. It aims to control the patient's effort during the game session in order to maximize his or her performance. It also includes the generation of game levels that are adapted to stroke patients. Second, we describe the design of PRehab, a game we developed for upper-limb rehabilitation. Finally, we study the effect of the proposed dynamic difficulty adjustment technique on patient performance, by comparing it with two control strategies, an incremental difficulty strategy and a random difficulty strategy.

The remainder of the paper is organized as follows: In Section 2, we review difficulty adaptation techniques in serious games for upper limb rehabilitation. In Section 3, we introduce the overall framework of the proposed adaptation approach. This includes the description of the design of PRehab, and an overview of the adaptation module. Section 4 focuses on the training module, which represents the main contribution of this paper. In Section 5, the experimental method is described. Section 6 presents the results of our statistical analysis. We discuss the results of the study in Section 7, and its limitations in Section 8. Finally, we summarize our findings and present our prospects for future work.

\section{State of the art}

The automatic adaptation of difficulty in video games has initially been referred to as dynamic difficulty adjustment. It has garnered the interest of both researchers and commercial game developers, as illustrated by the games MaxPayne (2001) and Left4Dead (2008). Automatic difficulty adaptation consists in adjusting the game parameters to the players' skills in real time. The objective is to create an optimal experience or flow, as first defined by Czikszentmihalyi (1991). The adaptation may be achieved through the modification of game scenarios, game world entities, difficulty levels, graphic user interfaces, etc. (see for example (Peirce et al., 2008; Conati et al., 2009; Chittaro et al., 2009; Rojas et al., 2012)). For instance, Hunicke et al. (2004) proposed an adaptive system called Hamlet, in which the strength of weapons, the virtual level of health, accuracy and other game entity properties are adapted to the player's skills. Hamlet is based on a probabilistic method to predict challenges for the players. Other techniques are based on the player's affective states and use motion and physiological data (Rani et al., 2005; Tijs et al., 2008; Kivikangas et al. , 2010). For example, Rani et al. (2005) proposed three difficulty levels that are based on the player's anxiety, as measured by physiological sensors.

Adapting the difficulty of a game may also be achieved via the game characters. For example, Spronck et al. (2006) developed a technique called dynamic scripting, which aims to control the behavior of computer-controlled opponents in computer role-playing games. It consists in adjusting the accuracy, the health points and the number of opponents of the player by maintaining 
several rulebases (one for each opponent type). The rules of an opponent are dynamically adjusted according to the success and failure rates of the player.

Recent studies have focused on the users' playing styles and skills to adapt game difficulty (Magerko et al., 2006; Zook et al., 2012). In these studies, the adaptation of the difficulty depends on the game's content, and in particular, on the scenario, the various levels and the game's narrative. For instance, Magerko et al. (2006) proposed an adaptation technique of the game story using a model of competence levels of healthy players. The adaptation of the difficulty consists in matching the game challenges with the player's gaming skills.

The objective of difficulty adaptation in serious games is not only to maintain the player's motivation but also to ensure the achievement of learning and training objectives. In the context of game-based learning and intelligent tutoring systems, this issue is referred to as the zone of proximal development (ZPD). These systems' objective is to keep students challenged and prevent them from getting frustrated or bored with difficult or repetitive activities (Wertsch et al., 1984; Murray et al., 2002). The techniques often depend on the students' situations and learning styles to adapt the game activity (McCuaig et al., 2012). For example, Conati et al. (2009) adapted the feedback of Prime Climb, a single-player educational game, on the basis of a student model that uses dynamic bayesian networks. In another example, Beynon et al. (2012) proposed the co-adaptation of a medical tutoring system, in which the patients could also adapt their skills. The authors used empirical modeling for medical education.

The goal of rehabilitation games is to improve the patients' training outcomes while maintaining their motivation during a therapeutic session. In this context, the difficulty of the game lies in the motor effort that the patient has to muster to achieve the game objectives. In order for the patient to regain his or her functions, this effort has to be maximized. Few studies have highlighted adaptation techniques for rehabilitation games. The first adaptation approach we found in the literature consisted in providing patients with adaptive visual, auditory and sensory feedback using virtual reality systems and robots (Varkuti et al., 2013). This approach is often taken in rehabilitation strategies that are based on music therapy (Markow et al., 2012), use specific training robots (Nef et al., 2012), and/or use augmented reality games to motivate patients with positive feedback (Khademi et al., 2012).

Since stroke patients generally have limited physical abilities, game difficulty has been deemed a critical adaptation target (Howcroft et al., 2012; Avila-Sansores et al., 2013). The difficulty of a task can be defined by the amount of physical effort necessary to perform it, or meet the proposed goal. It should be adapted to the patient's day-to-day abilities and health conditions. This means not only assisting the patient when the task is very difficult, but also challenging him or her to improve training outcomes (e.g., motion control, movement amplitude, accuracy and eye-hand coordination).

Next, we review various difficulty adaptation techniques in serious games for upper-limb motor rehabilitation. We first describe our analysis framework, 
and then present selected works. Finally, we conclude this section by discussing the results of our analysis.

\subsection{Analysis framework}

Our analysis uses the following criteria:

- What is the objective of the adaptation technique? This criterion provides information on the subject of the study and on its findings. The subject of the study may elicit the proposed difficulty adaptation technique and the required inputs.

- When are adaptation decisions made? This criterion assesses whether the decision on adaptation is made when playing (online) or before and/or after playing (offline). This also includes whether setting the difficulty level requires human intervention or not (i.e., it is automatically adjusted by the system).

- What are the inputs for the adaptation process? This criterion evaluates the data used to adjust game difficulty. Inputs can impact the decision on how and when the difficulty may be adapted to a given player.

- What are the game elements affected by the adaptation process? This criterion presents the results of the game adaptation. Specifically, it identifies the game elements used to adapt the difficulty.

- What is the model used for adaptation? This criterion determines whether the decision on difficulty adaptation is based on a specific model or on an ad-hoc solution (implicit). It provides indications about the re-usability of the technique in other rehabilitation games.

\subsection{Related work}

The first adaptation approach we found in the literature reviewed an offline adaptation of game difficulty. Annett et al. (2009) worked on Drumhab, a single-player music game based on a multi-touch laptop system called Ammi Interactive Rehabilitation Touch. This game aims to enhance eye-hand coordination and dexterity. The player was asked to perform pointing tasks that were synchronized with the music. To do so, the player had to use his or her hands as drumsticks, and hit the target drum to score points. The adaptation inputs were made up of data on the player's performance. Specifically, they included time-on-task, final player's score, number of touched beats, number of false hits, and beat touch accuracy. Game difficulty was adapted by the therapist before the playing started. The therapist selected the targets (drums) and set the game parameters, especially the number and the speed of the beats. One of the limitations of this difficulty adaptation technique is that it requires human intervention. Therefore, it prevents the therapist from supervising several 
patients simultaneously and/or remotely. In Annett et al.'s study, the evaluation of the approach was performed through interviews with patients and therapists, but focused only on the game's usability and acceptability.

Cameirao et al. (2010) highlighted a dual-task rehabilitation gaming system that used a computer, a camera and two data gloves. This system consisted of an adaptive rehabilitation game called Spheroids, in which players had to move virtual arms to intercept animated spheres. The objective of the authors was to study the effects of difficulty adaptation on task performance. The difficulty was automatically adjusted during the calibration of the system, by assessing the players' motor abilities: they were asked to move their hands to numbered dots located in specific locations on the tabletop. For each game trial, each hand and target position were randomly defined. The inputs for difficulty adaptation were based on performance and included speed and range of movement (combined shoulder and elbow aperture for arm extension) as well as latency (time to initiate a movement from a start cue). The difficulty of pointing tasks was adapted by adjusting the speed of the spheres, the interval between the appearance/dispersion of consecutive spheres. This technique was based on a psychometric model that uses experimental data to infer the relationships between game parameters and difficulty. It also takes previous player performances into account. However, the model is dependent upon the Spheroid game metrics and the calibration via predefined target positions.

Rabin et al. (2011) proposed an adaptive serious game for upper-limb rehabilitation. The objective of their study was to highlight the positive effects of serious games on motor control and shoulder/grasp strength. The game difficulty was based on an implicit model inspired by an incremental difficulty strategy. In other words, in a game session, the difficulty was progressively increased from easiest to most difficult. It was set at the start of each game session and for each player by a motor assessment exercise. In this assessment exercise, the player was asked to move his or her impaired arm as far as possible in different directions of a table. Accordingly, the following inputs were recorded for adaptation: reached zone, wrist weight, grasp pressure and session date. The difficulty could be increased during the game session by making the target area progressively smaller and by adjusting the speed and the range of the targets.

A key limitation to automatic difficulty adaptation is that it prevents therapists from adjusting game parameters. This, in turn, undermines their ability to modify their therapeutic strategies. To overcome this challenge, further works have suggested the use of a semi-automatic difficulty adaptation approach (Pirovano et al., 2012; Mihelj et al., 2012).

For example, Pirovano et al. (2012) used an intelligent game engine to adapt the games. These games could be played on Nintendo's Wii Balance Board and Microsoft's Kinect in the context of home rehabilitation. The system's objective was to provide upper and lower limb training by suggesting an adequate level of challenge. For instance, in Fruit Catcher, the player had to use a basket to catch fruits which were falling from the top of a tree. The difficulty was adapted by adjusting the number of falling fruits, number of 
baskets, fruit size, fruit weight and fall frequency. The parameters for each session were set on the basis of the player's performance in the previous session. The authors proposed a semi-automatic approach that allowed the therapists to monitor several system parameters that were independent from the game. Hence, the therapists could configure the system by selecting the exercises, the difficulty level, the time allocated for each exercise, the number of repetitions and the constraints on movements. This real-time adaptation was based on a quest bayesian model predicated on patient performance (i.e., the hit ratio in this game). This model was built with empirical data gathered from the performances of healthy players. However, stroke patient performance can vary widely depending on these patients' health condition. Thus, in order to be further validated, this model should also integrate stroke patient performance data.

Mihelj et al. (2012) proposed a virtual rehabilitation environment based on visual, sound, and haptic modalities. The objective of this work was to increase the motivation of stroke patients by focusing on game design. This technique aimed to provide patients with adaptive and meaningful game tasks by means of a story narrative. The scenario of the game unfolded through various levels (islands). On the computer screen, the players controlled a bottle and had to place it into a basket. The adaptation technique was based on several inputs. They included (i) task performance data: selected difficulty level, percentage of bottles caught and of bottles placed into the basket, and (ii) biomechanical measurements: mean absolute velocity and acceleration, total work and mean frequency of signal's position, velocity, acceleration and force. The difficulty levels were predefined by adjusting the speed and the range of the bottles and of the basket. The adaptation process was based on an incremental difficulty strategy. Though quite intuitive in its approach, this adaptation remained based on player preferences gathered from previous game trials. These preferences were integrated to adjust the game difficulty by mapping user performance and game inputs using a stepwise linear discriminant analysis.

\subsection{Discussion}

The online difficulty adaptation approach has garnered the interest of many researchers in rehabilitation games (Cameirao et al., 2010; Rabin et al. , 2011; Pirovano et al., 2012; Mihelj et al., 2012). This approach aims to automatically adapt the game, which allows the therapist to remotely and simultaneously supervise several patients. It encompasses two current difficulty strategies: (i) dynamic difficulty adaptation, in which difficulty is adjusted (i.e., increased or decreased) during the game (Cameirao et al., 2010; Pirovano et al., 2012); and (ii) incremental difficulty adaptation, in which difficulty is progressively increased during the game session (Rabin et al. , 2011; Mihelj et al., 2012). Though most studies have attempted to show the usefulness of games for improving training outcomes e.g., motor control and shoulder/grasp strength(Rabin et al. , 2011) 
as well as their usability and acceptability (Annett et al., 2009; Pirovano et al., 2012), they have failed to consider the effect of a given difficulty strategy on patient performance.

In the above-reviewed studies, the player's profile was often implicit and defined by the training metrics. The adaptation inputs mainly consisted in (i) the player's performance, such as his or her final score (Pirovano et al., 2012), time on tasks (Annett et al., 2009), movement accuracy (Cameirao et al., 2010), zone reached, wrist weight, grasp pressure (Rabin et al. , 2011) and (ii) biomechanical measurements, such as the mean frequency of signal's position and force (Mihelj et al., 2012).

Adaptation parameters vary with the objective of the rehabilitation program and the space and time constraints of the therapy. The goal of rehabilitation may be to improve (i) training volume, e.g., the number of tasks and the time elapsed game session (Annett et al., 2009; Pirovano et al., 2012) and (ii) training quality, e.g., the range, accuracy and speed of movements (Annett et al., 2009; Mihelj et al., 2012). In our analysis, we identified two sets of parameters: (i) system parameters, e.g., the time allocated to each exercise, number of repetitions and the difficulty level (Annett et al., 2009; Pirovano et al., 2012) , and (ii) game metrics, e.g., the number, range and speed of targets (Annett et al., 2009; Mihelj et al., 2012) and the interval between the appearance/dispersion of targets (Cameirao et al., 2010). Adaptation techniques often share similar game scenarios, in which only the game metrics can be adjusted. However, stroke rehabilitation programs are based on long-lasting repetitive tasks, which can alter patient motivation and commitment.

Finally, in these early attempts, the game adaptation model was often implicit (Annett et al., 2009; Rabin et al. , 2011). Until recently, most adaptation techniques employed ad-hoc solutions and thereby lacked reusability. Lately, empirical data and probabilistic models have been used to refine the difficulty adaptation model. To make difficulty adjustment decisions, these techniques use various approaches, such as psychometric modeling of experimental data (Cameirao et al., 2010), bayesian modeling (Pirovano et al., 2012), and stepwise linear discriminant analysis (Mihelj et al., 2012). Nevertheless, in spite of their valuable contributions, these models still depend on specific game metrics, and therefore lack reusability.

Instead, we propose a dynamic difficulty adaptation technique based on the player's profile. To be generic, this technique focuses on the generation of pointing tasks, which difficulty can be dynamically adjusted. The adaptation technique seeks to control the patient's effort during the game session in order to maximize his or her effort while preventing fatigue. It also offers the generation of game levels that are dynamically customized based on the difficulty strategy. 


\section{The overall framework}

In this section, we describe the overall framework of the proposed system. First, we discuss the various design steps of a rehabilitation game. Then, we present PRehab, a game we developed specifically for upper-limb rehabilitation purposes. Finally, we review the adaptation server used to adapt the game's difficulty and to generate levels in PRehab.

\subsection{Game, tasks and objectives}

Post-stroke rehabilitation is often a long process, lasting many months or even years. Patients should undergo multiple-session therapy in order to regain their functions. Rehabilitation games should be considered a component of the overall rehabilitation protocol, and not a self-sufficient activity. Moreover, since rehabilitation is a long process, the challenge for game designers is to create games which can generate patient excitement and sustain their motivation throughout the entire duration of the therapy.

Several studies have focused on the design of virtual reality systems and serious games for stroke rehabilitation (Goude et al., 2007; Burke et al., 2009; Mihelj et al., 2012). The design of a rehabilitation game is critical in that it must meet the application domain requirements while providing the player with an enjoyable experience. It can influence game rules, win and failure conditions, game mechanics, ambiance, scenario and all the game content elements which relate to the rehabilitation requirements. We employed a user-centered approach, which may be considered a tenet of good practice for game design. It consists in involving end users (i.e., patients and therapists) in the design process to ensure that the game precisely meets their needs. This process is iterative; it involves usability tests at the end of each design iteration.

Various constraints have to be taken into account when designing a rehabilitation game. These constraints vary with the objectives of each session. For example, in upper-limb motor rehabilitation, time constraints are important because patient fatigue has to be prevented. For example, in this context, a typical game session duration could be set to 20-45 minutes per day. Additionally, the game should be played every day for at least three months. Thus, a successful game has to maintain patient motivation and commitment throughout this long period. To achieve this, we decided to structure our game into several sections inspired by classical theatre units based on "acts" and "scenes" (Sherlock, 2005). The game is considered a complete unit defined by a goal. To reach this goal, players have to go on an adventure and reach several intermediate objectives through a series of acts.

Acts correspond to game levels. They comprise one or more scenes. Each scene is the current view of the world during the player-game interaction. This dependence between the goal and the intermediate objectives seeks to maintain a logical link between the various acts, which may be played during different sessions. 
For example, the goal of the famous, classic Super Mario game is to free Princess Daisy. To achieve this goal, Mario and his brother Luigi must fight and defeat various enemies at each game level. Indeed, each level defines its own universe, illustrating the concept of a classical theatre act. In Super Mario, each act features a primary scene in which the enemies are located, and secondary scenes reached through special pipes. The main characters' moves and actions (e.g., running and jumping) are designed to make them achieve the objectives (i.e., to eliminate enemies). Meeting intermediate objectives helps the player advance toward the final goal, which is the liberation of Princess Daisy.

In a rehabilitation context, this structure can be used as follows:

- The game defines the goal to be reached by the patient at the end of the rehabilitation program. We expect the lifetime of the game to have the same order of magnitude as the approximate length of a post-stroke rehabilitation program (3 to 12 months).

- Each game act/level corresponds to a training session of 20 minutes. This duration can be set by the therapist at the beginning of the session. To promote a sense of achievement and self-esteem, it is important to set an explicit and achievable goal for each level.

- Each game scene represents the stage upon which the patient plays the game as therapy, by carrying out pointing tasks.

A pointing task is defined by a target and a starting point where the hand is initially positioned. To reach the target, the user has to move the "cursor" from the start position to the target position without clicking. The pointing task parameters (e.g., target position and range, initial hand position) combine with the patient's abilities to define a level of difficulty that determines success or failure. Successfully performing a pointing task depends on one's ability to reach the target within specific time and space constraints. In early stages of rehabilitation, however, no constraints are placed on patients, who are merely asked to reach the target. In subsequent rehabilitation stages, depending on the patient's abilities and health condition, the training difficulty may be increased to enhance the quality of movements. For example, time and space constraints (e.g., to quickly reach the targets) may be added.

\subsection{PRehab}

We designed and developed PRehab (Platform games for Rehabilitation), a rehabilitation game that can be played on a graphics tablet (for more information, see our video presentation in (PRehab, 2014)). This game was designed for upper-arm rehabilitation through pointing tasks (i.e., reaching targets). Using the mouse of a graphics tablet, the patient has to reach virtual targets. The therapeutic objective is to increase the range of motion as well as the training volume (i.e., number of tasks) during the rehabilitation session.

The ultimate goal in PRehab is to protect Nature by collecting all of the gemstones. Each game is composed of a set of levels generated during the game 


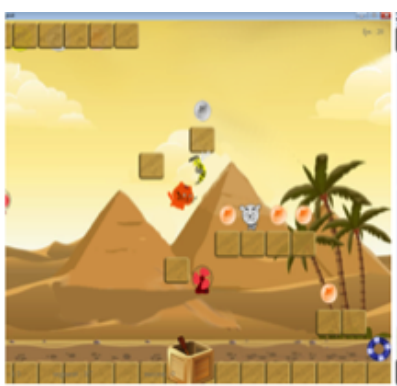

(a)

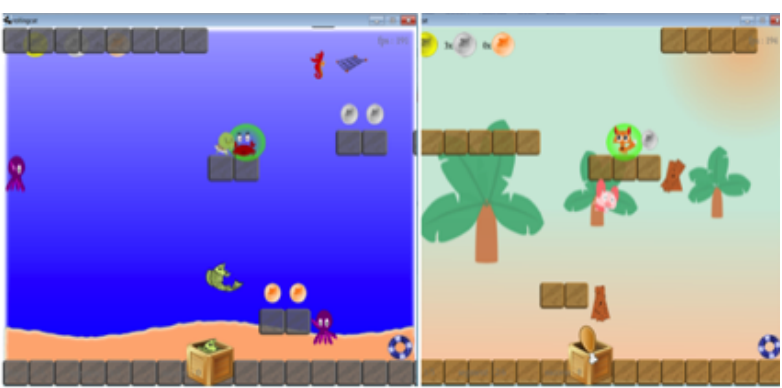

(b)

(c)

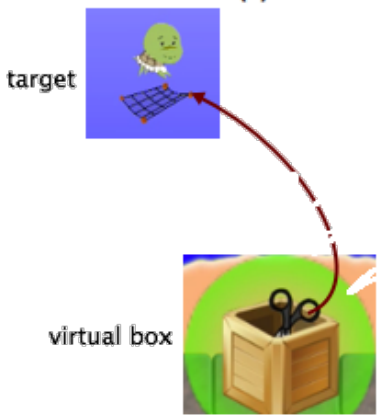

(d)

Fig. 1 Game environments implemented by PRehab. Graphics change but mechanics remain similar from one environment to the other: (a) Desert: Cat game, (b) Sea: Turtle game and (c) Forest: Rabbit game. (d) Reaching a target with an object in the virtual box.

session. The player controls the game's main character, and has to help him reach the end of each level. A level is composed of a set of scenes which unfold along the player's progression. At each level, the objective is to eliminate enemies in order to reach the gemstone. Enemies act as obstacles in the main character's progression. Additionally, the player can collect various coins to increase his or her score.

Each game level scene is composed of (i) blocks (connectors) that direct the main character's movements, and (ii) enemies who block the way. To free the character's way, the player has to use objects from his or her virtual box to eliminate obstacles (as illustrated in Fig. 1 (b) and (d)). From a motor training perspective, the game's pointing tasks require that players reach targets (i.e., enemies) which appear in different areas of the game scene. A pointing task is performed successfully when the target is reached. When the player fails to reach the target, the main character is blocked and a time limit prevents deadlock. At the end of the time limit, the main character automatically moves on to the following scene.

To encourage players to perform actions (i.e., movements) requiring greater amplitude, we assigned two different values (gold and silver) to the reward coins: gold coins are obtained upon performing the most difficult actions, while silver coins reward the less difficult ones. This decision was made following a 


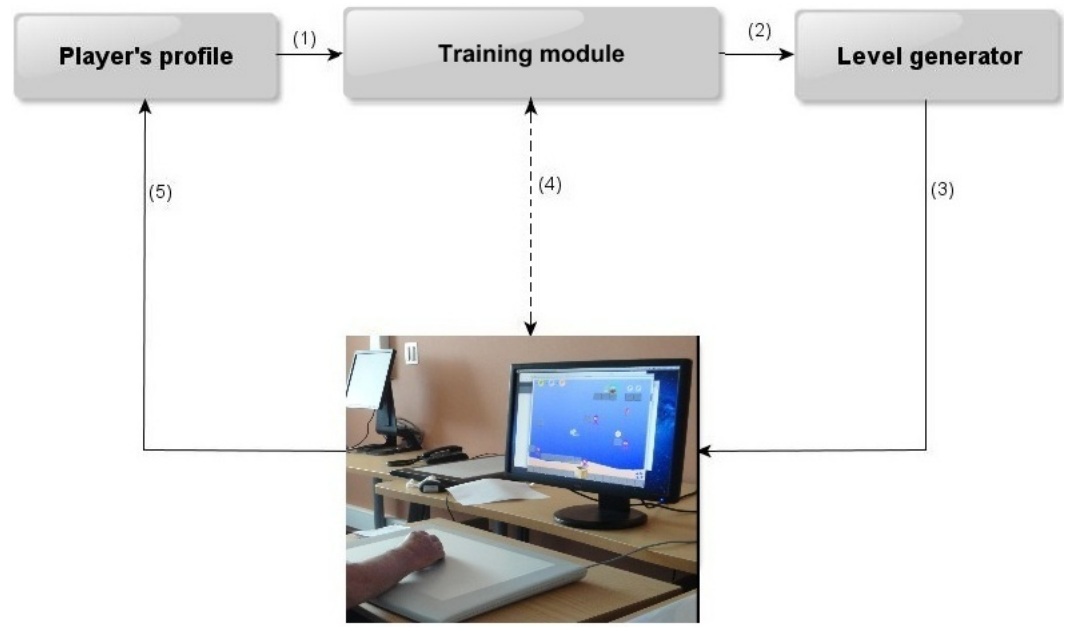

Fig. 2 Adaptive system architecture.

test-play session with game developers and gamers, who helped us determine which potential elements would increase the game's fun factor.

Finally, because rehabilitation requires repetitive tasks which may induce patient boredom, it is preferable to have a large number and variety of games. From a game development perspective, our approach when designing PRehab was to clearly distinguish the game mechanics from its graphics. In other words, once the rules and behaviors of game entities are implemented, they are then reused in other games with different graphic environments (or aesthetics), as illustrated in Fig. 1: (i) in the Sea environment, the main character is a turtle (Turtle game); (ii) in the Forest environment, a rabbit character (Rabbit game); and (iii) the Desert environment, a sand cat (Cat game). The three game environments retain the same mechanics and behaviors. The map, the various entities (enemies, objects) and the main character use different graphics (i.e., images and animations).

In PRehab, the games are adapted using an adaptation server. The system is based on a client/server architecture. The client represents the game instance for a given patient. The server is responsible for tracking patient movements, adapting game difficulty and generating customized game levels.

\subsection{Adaptation server}

The proposed adaptation server is independent from the rehabilitation game. Its internal architecture is shown in Fig. 2.

The adaptation process can be summarized as follows: the training module generates adaptive pointing task sequences (2) on the basis of the player's profile (1); the procedural level generator module uses these sequences to generate a game level (3); when the level is played, the difficulty is dynamically adapted 


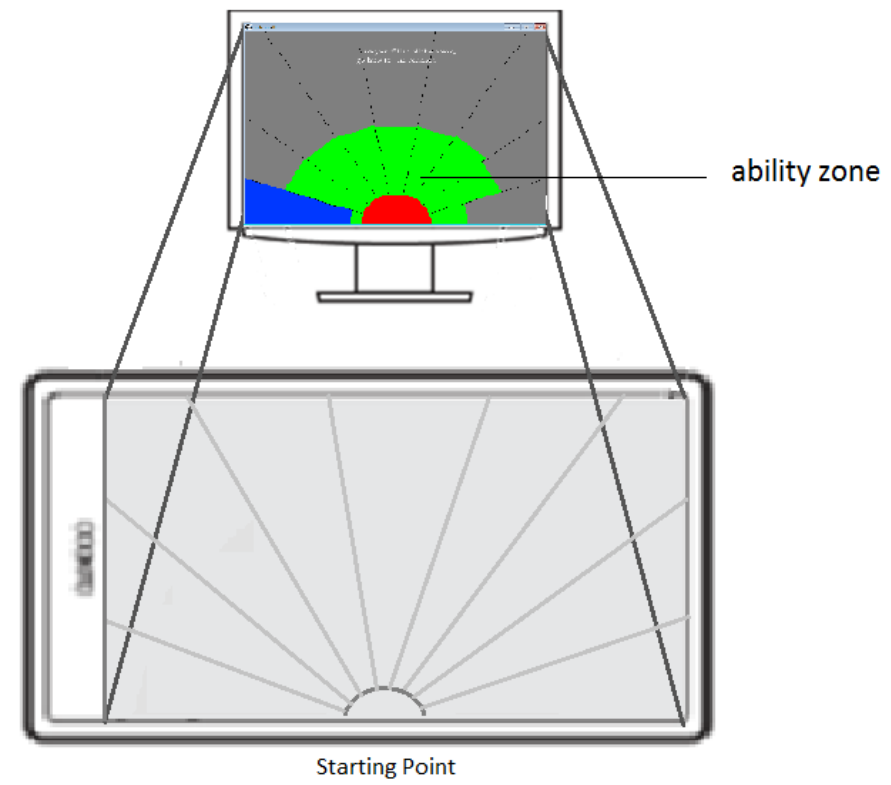

Fig. 3 Assessment exercise: the patient is instructed to cover the entire workspace by moving into 9 different directions.

to the player's performance (4); the player's performance is then integrated, and used to update the player's profile (5). Next, we introduce the main modules of the adaptive system. In particular, we review the player's profile, the training module and the game level generator.

\subsubsection{Player profile}

Automatically adapting the difficulty in a rehabilitation game requires a computational model of the patient's motor abilities. The proposed model is based on the short-term prediction of the player's abilities and the daily physical condition of the stroke patient. This is critical, because stroke patients typically exhibit a slow recovery rate and their performance varies with their daily health condition.

The training module requires the assessment of the player's abilities, known as the "ability zone" (Hocine et al., 2011). The ability zone represents the area where the patient can effectively make movements on a $2 \mathrm{D}$ workspace (e.g., a graphics tablet). In early stages of rehabilitation, stroke patients may be unable to reach the targets farthest from the starting point position (see Fig. 3 ), or targets on a particular side of the workspace.

The workspace is divided into a several areas and represented by the ability zone. This ability zone is modeled using a matrix of dimension $n \times m$, where $m$ represents the number of rows and $n$ the number of columns. This matrix constitutes the mapping between the physical workspace (e.g., $1 \mathrm{~m} \times 1.50 \mathrm{~m}$ 
calibrated at the start of the therapeutic session) and the virtual workspace (computer screen). Each matrix cell holds information about the movement performed by the patient in the corresponding zone of the workspace. To assess his or her abilities, the patient has to move the mouse of the graphics tablet in various directions within the workspace. The system uses the mouse coordinates to compute the ability zone of the patient.

In (Gouaich et al., 2012), we proposed a bio-inspired method to build the ability zone matrix. In this method, a virtual ant (i.e., located on the screen) follows the hand movement of the player on the workspace. When the patient performs a task, the ant secretes pheromones. Following this model, the cells of our matrix contain digital pheromones that indicate the extent to which the corresponding areas of the workspace have been reached by the patient. Using the evaporation law of pheromones, we can ignore any region which has been reached by chance because of uncontrolled movements. In addition, the propagation law of pheromones allows us to study the accuracy of movements according to the patient's recovery stage.

The ability zone is defined in the assessment exercise, and updated during the playing session. In this exercise, the patient is asked to cover the entire workspace by moving the mouse into 9 different directions, as shown in Fig. 3. This data is then used to define the initial ability zone of the patient. Further in the adaptation process, this data will also help identify challenge and assistance areas.

\subsubsection{Training module}

Our difficulty adaptation technique relies on the generation of pointing tasks according to the player's profile. It is based on heuristic search, specifically a Monte Carlo tree search algorithm (MCTS). This MCTS algorithm performs simulations to find the best decision among a random set of solutions. In PRehab, we used it to compute the best pointing task sequences, so as to create several difficulty modes in the game. A detailed description of the training module is given in the next section.

\subsubsection{Game level generator}

To diversify the game content, the adaptation process relies on the real-time generation of game levels. We therefore examined procedural content generation (PCG) techniques which enable the automatic creation of content through algorithmic means (Togelius et al., 2010; PCG, 2014). In general, PCG techniques have been used to develop low-cost resources to diversify game content. Different game elements can be generated, such as game world entities, terrain, maps, levels, stories, scenarios, quests and rulesets. We focused on game level generation in platform games for rehabilitation. In PRehab, procedural content generation is used to create game scenes by selecting adapted and coherent game entities. 
The proposed approach consists in generating the game scenes that constitute the level while ensuring their playability. The pointing task sequences provided by the training module serve as inputs for the generation of the scenes. These sequences are used to determine the targets' position in each scene of the level. The next step consists in "filling the gaps" between the targets by adding connectors to insure the player's progression. Connectors are game entities used to direct the main character's movements.

The level generator creates a level description file using a grammar representation. This file is communicated to the game engine in order to create the game level. The level description file includes the description of the game entities of each scene constituting the level. In particular, game entities such as targets (enemies), connectors, as well as the player's own entity are described by their positions in the game scenes.

\section{Detailed description of the training module}

The proposed adaptation technique involves the generation of the scenes of each game level according to the player's profile. The training module generates the lists of pointing tasks and provides them to the level generator. Since this process could take a long time and might not be compatible with interactivity constraints during the game session, the generation of pointing tasks is performed before the play (offline). The game scenes are generated for each difficulty mode and the transition between scenes is based on the player's performance (online).

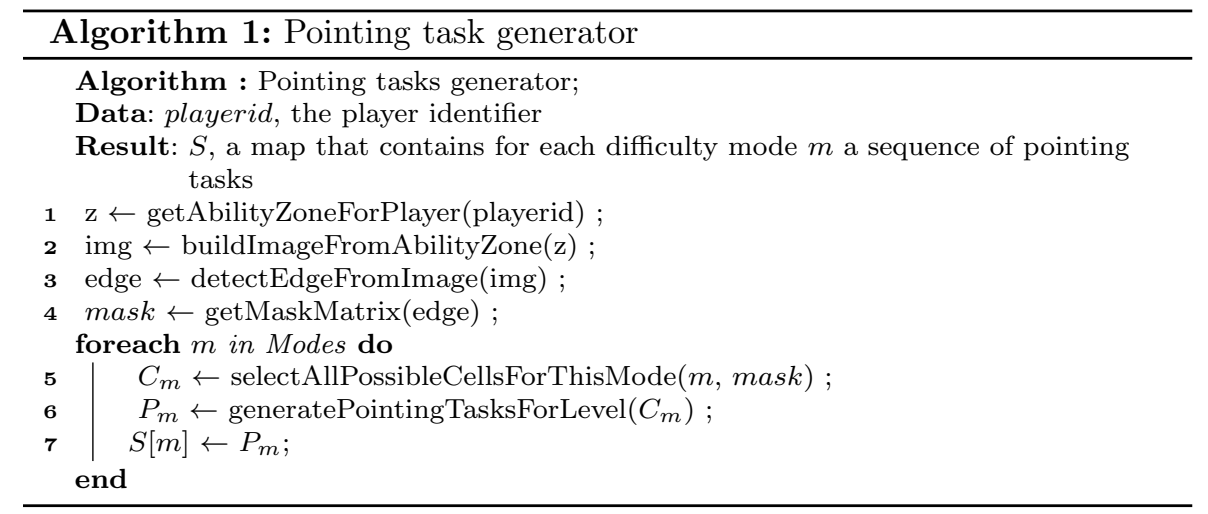

The pointing tasks generator is presented in Algorithm 1. This algorithm uses the player's identifier playerid as an input to retrieve his or her current ability zone (line 1). The ability zone image obtained is then used to determine the areas of the workspace which are difficult or easy for the patient to reach (lines 2 - 4). In particular, the edge of the ability zone image is used to compute 
the mask matrix that allows the training module to determine the difficulty of pointing tasks.

For each predefined difficulty mode, a list of all potential areas is computed (line 5). This list is filtered using heuristic search defined in Algorithm 2. This algorithm is based on a Monte Carlo tree search which selects the "best" pointing task sequence for a game level (line 6). Next, we describe the main steps of this algorithm.

\subsection{Step 1: generating pointing tasks per difficulty mode}

Increasing training volume (i.e., number of tasks) and range of motion is an important objective in upper-limb rehabilitation strategies. This can be achieved by asking the patient to reach several targets which appear as far as possible from the starting point of the workspace. The difficulty of each pointing task depends on the movement direction and on the distance between the starting point and the target.

The training module uses the ability zone of the patient to compute the difficulty of pointing tasks. Since the ability zone is a matrix in which each cell contains the digital pheromone's intensity, we can build an image representing this matrix where the pheromone is translated to a grey scale color.

The edge of this image represents the farthest area that the patient has reached. Thus, to design adapted challenges, we integrate the patient's initial abilities. We assume that these challenges will have a sustainable training effect that will increase the patient's range of motion.

To detect the edge of the ability zone image, we use a Sobel operator (Vincent et al., 2009). The Sobel operator is an image processing, edge detection algorithm used for images with high frequency variations. The operator emphasizes regions of high spatial frequency that correspond to edges. It is based on a pair of $3 \times 3$ convolution kernels $k_{x}=(-1,0,1 ;-2,0,2 ;-1,0,1)$ and $k_{y}=(1,2,1 ; 0,0,0 ;-1,-2,-1)$.

These kernels are used to compute the gradient of each pixel of the image vertically $\left(G_{x}\right)$ and horizontally $\left(G_{y}\right)$.

These measurements are then used to compute the gradient magnitude on the basis of the following formula: $|G|=\sqrt{\left(G_{x}\right)^{2}+\left(G_{y}\right)^{2}}$.

With the gradient obtained, we compute the mask matrix $(A)$ for the training module to determine the challenge and assistance areas:

$$
A(i, j)=\left\{\begin{array}{l}
\text { true if }(G(i, j)>t) \\
\text { false otherwise }
\end{array}\right.
$$

Where $t$ is a threshold parameter that determines the extent to which the movement frequency is considered. The training module uses this mask matrix to determine potential targets in different difficulty modes. Five difficulty modes are defined: easy, medium, difficult, very difficult and expert. These modes are based on space constraints, and particularly the distance between the hand and the target, as well as the movement direction. 
More specifically, these modes are designed as follows:

- Easy mode: The target is placed very close to the starting point.

- Medium mode: The target is placed as close to the bottom image edge as possible. The objective is to decrease the difficulty so that the patient may be able to reach the targets.

- Difficult mode: The target is placed in the farthest area reached by the patient, i.e., the edge of the ability zone image defined by the Sobel operator.

- Very difficult mode: The target is placed outside of the ability zone.

- Expert mode: The target is placed far beyond the edge of the ability zone image.

\subsection{Step 2: selecting pointing tasks for a game level}

The first step of the process creates a mapping that links each difficulty mode to the areas of the workspace, where pointing tasks with equivalent difficulty can be generated. Since the number of potential pointing tasks is very large, we must find a way to select a few for each level. In (Gouaich et al., 2012), we used a random selection of pointing tasks. We found that this selection method often results in poorly-defined game scenes, in which symmetry and distribution rules are not taken into account. For example, a random outcome may cluster all the targets in one place, which produces a graphically imbalanced game scene. It can also influence the playability of the game.

Moreover, from a training perspective, using a random selection can generate targets which may be too easy to reach for the patient. This runs contrary to the usual rehabilitation approach, which aims to progressively lead patients to greater movement amplitude by decreasing the difficulty of tasks so they are able to perform them. This approach ensures that patients move in different directions within the workspace, so as to consistently increase their range of motion.

To meet this key requirement, we decided to base the target selection method on a Monte Carlo Tree Search (MCTS) algorithm. The objective was to generate the most suitable sequence of pointing tasks, taking both training and gaming constraints into account.

MCTS seeks optimal decisions by building a search tree from random samples in the decision space (Tavener et al., 2012). Its general algorithm involves four steps:

- Selection: Starting from a root node, the selection aims to find the optimal child node that maximizes some quantity until a leaf node is reached.

- Expansion: In this step, one or more child nodes are added to expand the tree according to available actions. Then, a child node $C$ is selected according to a tree policy.

- Simulation: A simulated playout is run from $C$ until a result is achieved.

- Backpropagation: The move sequence is then updated with the simulation results according to a default policy. 
These steps can be grouped into two policies: (i) a tree policy to select or create a leaf node within the search tree (selection and expansion) and (ii) a default policy that produces a value estimate (the simulation of the action).

The pointing task generator builds five search trees according to the difficulty modes. Each tree node represents a state of the game level which is a pointing task. The node contains information about the rewards as well as the number of visits to this node.

The search consists in iteratively building a tree until a budget is reached. The latter represents: (i) the given time to find a solution (number of iterations); and (ii) the volume (i.e., number of tasks) of the level.

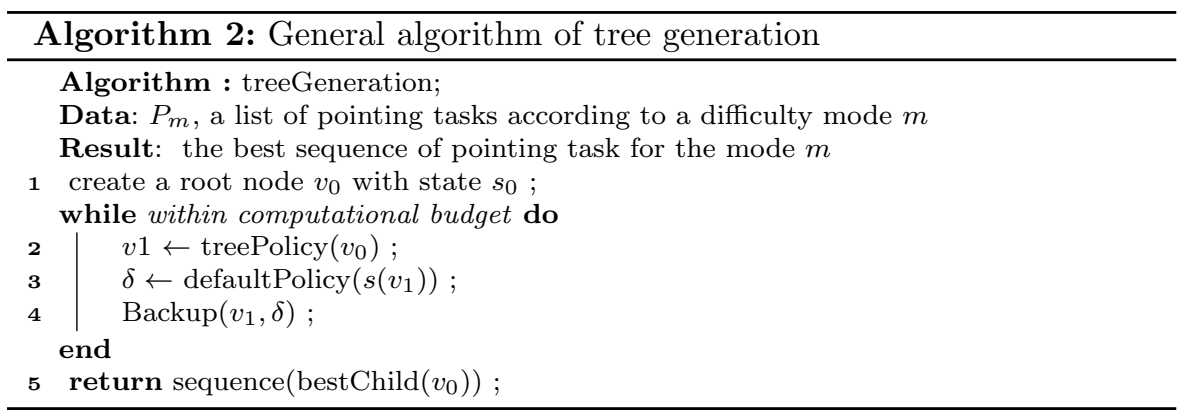

The pseudo code of the tree generation is given in Algorithm 2. This algorithm uses a list of pointing tasks in a difficulty mode as an input. The first step of the tree search consists in selecting a root node $v_{0}$ which represents the initial target in the state $s_{0}$ (line 1). Then, within the computational budget, the algorithm iteratively builds the tree. The tree policy aims to select a leaf node (line 2). The default strategy is run to simulate the new node (line 3 to 4 ), where $\delta$ is the reward for the last reached state. The result of the overall search (line 5) is the sequence of all the best nodes selected by the search.

The tree search produces a sequence of pointing tasks selected to maximize the reward function. This consists in selecting the best node (i.e., a target) on the basis of the Upper Confidence Bounds for Trees (UCT) method (Kocsis et al., 2006). The best node is selected according to its UCT value given by the following formula:

$$
V=\overline{R_{j}}+\sqrt{\frac{2 \cdot \log (n+1)}{n_{j}+\epsilon}}
$$

Where $\overline{R_{j}}$ is the average rewards from the node $j$ that are understood to be within $[0 ; 1], n$ is the number of times the current parent node has been visited, $n_{j}$ is the total number of times that the child node $j$ has been visited and $\epsilon$ is a tunable bias parameter. 
In this formula the reward term $\bar{R}_{j}$ encourages the exploitation of nodes with higher rewards and the right-hand term $\sqrt{\frac{2 \cdot \log (n+1)}{n_{j}+\epsilon}}$ encourages the $e x$ ploration of less visited choices. UCT ensures the property of equilibrium between exploitation and exploration. To develop the ability zone of patients, it is important to provide them with tasks that increase the reward function while checking their ability to explore the workspace.

The reward value $R$ for a node $j$ is given by the following formula:

$$
R=\frac{1}{2}(\alpha . \operatorname{distance}(j)+\beta . \operatorname{shift}(j))
$$

Where $\alpha$ and $\beta$ are used to encourage the playability factor $(\alpha+\beta=1)$. The reward function depends on two factors:

- The usefulness of the solution: It is the importance of reaching a target in a particular location within the workspace. The usefulness is represented by the function distance $(j)$, which is the ratio between: (i) the distance from the starting point of the workspace to the child node $j$ and (ii) the maximum distance between the starting point and the children nodes.

- The playability value of the level: This value is given by the function $\operatorname{shift}(j)$ that determines the extent to which the child node $j$ is aligned to the left in the game scene. This allows the generation of scenes that contain a variety of targets.

The training module thereby allows the online adaptation of difficulty by suggesting adapted pointing tasks to the patient during the game session. It does so by selecting the most appropriate difficulty mode according to the patient's performance. Specifically, the player's success and failure rates are used to make decisions about increasing or decreasing the game difficulty.

In addition, the therapist has several ways to control the session. He or she may select the game and the number of targets proposed to the patient at each level. The therapist may also update the parameters of the ability zone in terms of the workspace dimensions and the movement accuracy. This may be useful in some situations (e.g., when the therapist needs to change devices and calibrate another system; or when the patient is at an advanced recovery stage, and expected to perform more accurate movements). Finally, the therapist can also get feedback on the patient's performance at the end of the game session.

\subsection{Example}

The dimension of the workspace and the matrix can be set at the start of the game session. In the experiment, we considered an ability zone of $30 \times 30$ to map the graphics tablet workspace with the screen. To explain this concept, we consider a simple example of an ability zone $(7 \times 7)$ shown in Fig. 4 (1).

The dimension of the workspace and the matrix can be set at the start of the game session. In the experiment, we defined an ability zone of $30 \times 30$ to 


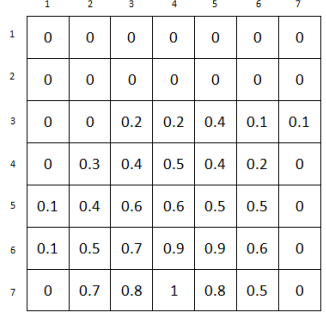

(1)

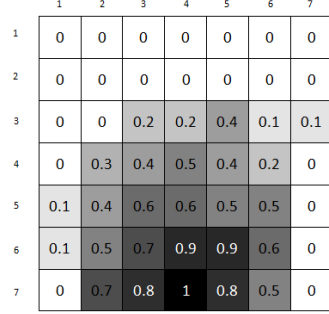

(2)

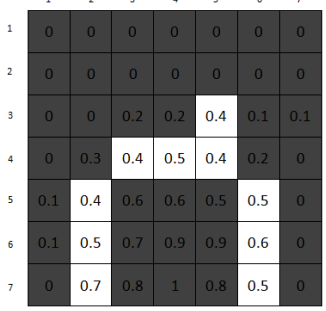

(3)

Fig. 4 Examples of an ability zone (1), an image obtained (2) and its edge (3).

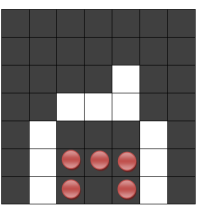

Easy

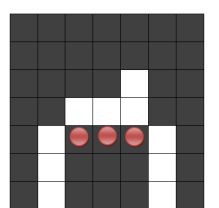

Medium

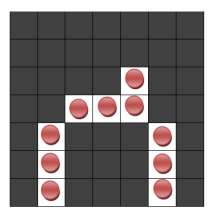

Difficult

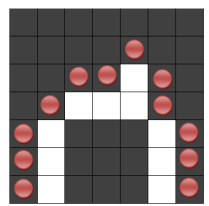

Very Difficult

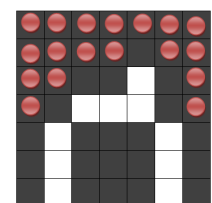

Expert

Fig. 5 Exemples of potential targets in a scene, according to difficulty mode.

map the graphics tablet workspace with the screen. To explain this concept, we offer the example of a $(7 \times 7)$ ability zone, as shown in Fig. 4 (1).

Using the digital pheromone-based algorithm (Gouaich et al., 2012), the ability zone is represented by a matrix in which each cells contains the normalized pheromones' intensity, to be understood within $[0 ; 1]$. The pheromones' intensity of a cell refers to the ability of the patient to reach targets in the corresponding area of the workspace. Using this matrix, an image is built by assigning a gradient color to each matrix cell (see Fig. 4 (2)). The edge of this image is then computed using the Sobel operator, given a gradient threshold of $t=0.4$ (see Fig. $4(3)$ ). This edge represents the maximum amplitude of the movements made by the patient.

The image obtained is then used to compute the mask matrix, which, in turn, yields information about the assistance and challenge areas. Potential target positions are generated according to the difficulty mode. Examples of potential target positions for each difficulty mode are shown in Fig. 5.

Then, for each difficulty mode, a search tree is built in order to obtain the best sequence of pointing tasks. For instance, Fig. 6 illustrates the sequences obtained for a level training volume of $v=8$ and a maximum number of iterations of 300 .

Fig. 7 shows an example of the game scenes proposed to the player. First, the default difficulty mode is difficult (1). As mentioned above, the objective of rehabilitation is to maximize the patient's effort. Only when the tasks are considered very difficult does the game decrease the difficulty. Our adaptation 


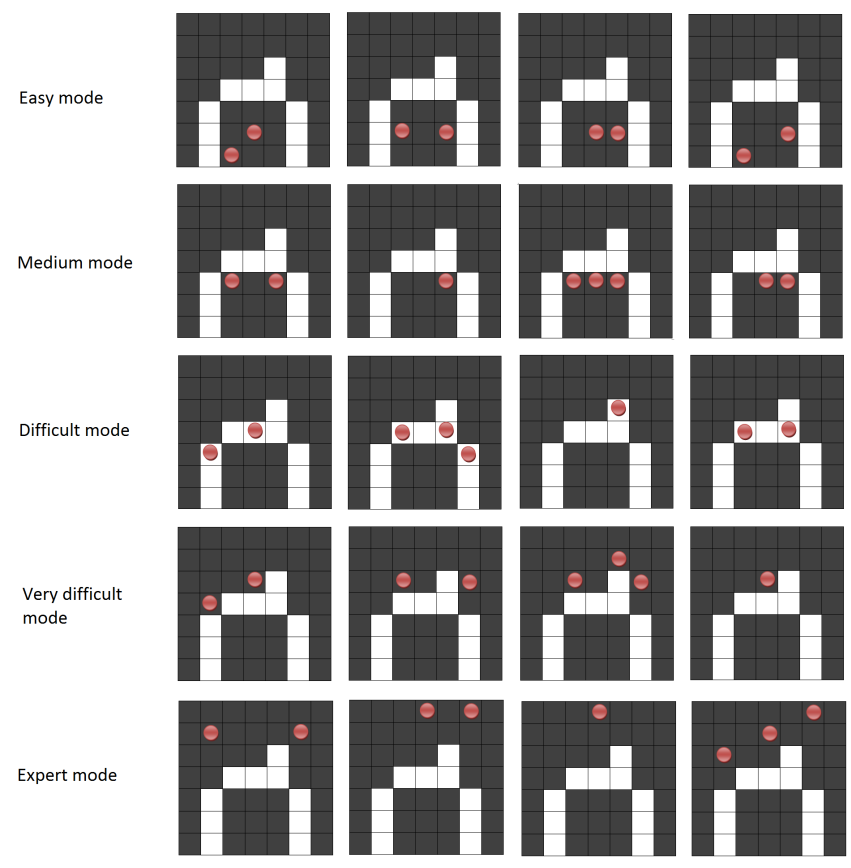

Fig. 6 Examples of pointing task sequences for each difficulty mode.

technique is based on this decision-making hypothesis. Moreover, the game may propose easy tasks to the patient, following $1,2, \ldots, \mathrm{n}$ successive failures.

The same decision process may apply in the case of consecutive successes. In this example, starting from a difficult scene, when the window of success and the window of failure is $w=1$, and if the player meets the scene's objective, the next scene will be a very difficult one (2). If the player does not meet the initial scene's objective, he or she is then assigned to a scene in the medium mode (3). The default value of this window is set to 1 in PRehab, because the game was initially proposed to stroke patients with severe physical difficulties. However, it can be configured by the therapist at the start of the game session to propose more challenging or easier game scenes to the patient based on his or her condition.

In PRehab, game levels can contain "floors" corresponding to the five difficulty modes. Fig. 8 shows a screenshot of a zoom on all five floors. At the start of the game level, the player's character is located on the floor corresponding to the difficult mode. The game difficulty is then adjusted on the basis of the player's performance. This consists in letting the player's character move on to the next scene, i.e., ascend to the floor above or descend to the one below. In this case, the adaptation focuses on camera movements to propose adapted game scenes to the player. 


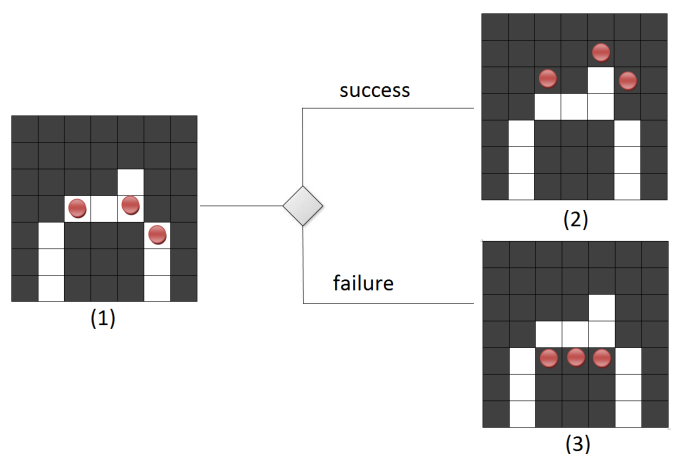

(3)

Fig. 7 Example of proposed scenes to a player.

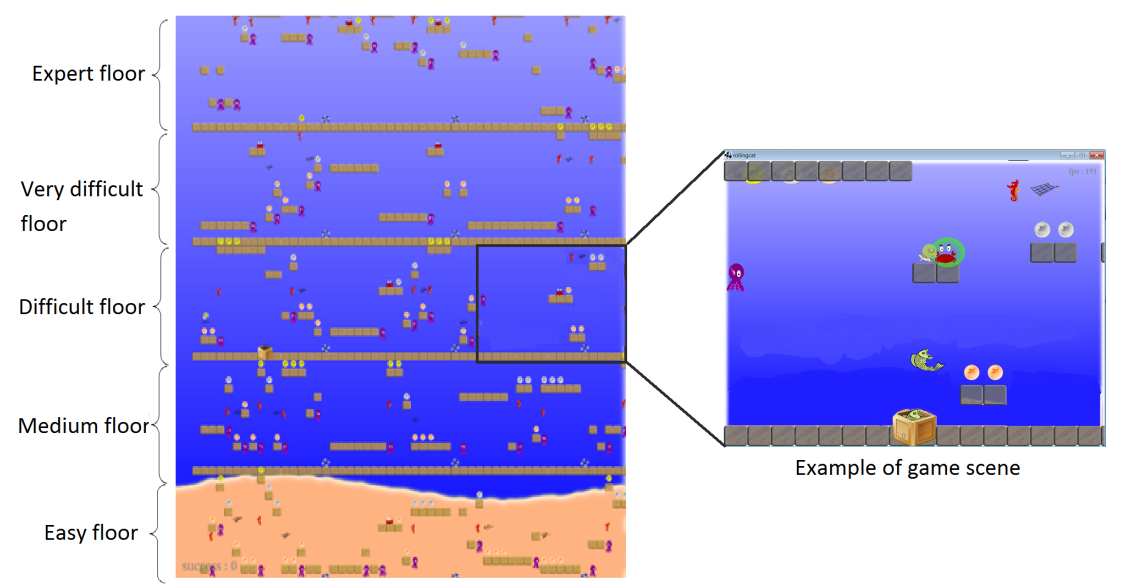

Fig. 8 Screen capture of a PRehab game scene (Turtle). The right image shows the scene as viewed by the player. The left image shows the entire multi-level game environment, in which the player's scene is unfolding.

\section{Method}

The objective of our experiment was to study the effects of the proposed dynamic adaptation technique on the training outcomes of patients. The study included several tests and was based on a participatory design process that involves physicians, therapists and stroke patients. Next, we describe the experimental design and protocol. 


\subsection{Experimental design}

We followed a design method that takes into account the practical issues related to the adaptability of rehabilitation games in stroke patients. This usercentered design process focused on the continuous evaluation of the system through its successive development phases. It is based on an iterative protocol requiring a usability test at the end of each game design iteration. Our study involved three types of evaluations: (i) a playtest with healthy players and medical experts (ii) a usability test to evaluate the adequacy of the graphic user interface for stroke patients and (iii) an experiment to assess patient performance in the different difficulty strategies. All tests were carried out with patients and therapists from the Lapeyronie Hospital of Montpellier (France) and Grau du Roi Hospital of Nimes (France).

The objective of the playtest was to evaluate the playability of PRehab. Eight healthy participants were selected. They included professional game developers and two experts in stroke rehabilitation. The players were asked to assume the role of patients and were explained the various implications of motor disabilities for stroke patients attempting to play a video games. Our main objective was to identify the game's bugs and to evaluate its fun factor. Each player evaluated the game individually and an observer was recruited to write the final report. Lastly, the two stroke rehabilitation experts also played PRehab and reported on it from their perspective.

The players' recommendations were essentially to add game feedback to increase patient motivation. We therefore added encouragement messages and refined the coin reward procedure by introducing silver and gold coins to further reward patient performance. We also realized that a tutorial was necessary and incorporated one at the start of the game. Furthermore, the stroke rehabilitation experts noted that patients with severe cognitive deficits would probably need assistance to see the targets. Thus, we decided to highlight the targets when a maximal reaction time threshold is exceeded.

After reviewing technical issues and discussing items mentioned by the experts, we conducted a usability test with an actual patient. The patient was 76 years of age, and presented with an impaired left arm. He was asked to play PRehab while being observed by five therapists. He first played the assessment exercise, and then played a PRehab version based on the proposed dynamic difficulty adaptation technique for 30 minutes. A questionnaire was administered at the end of the session to evaluate the patient's perception of motivation, challenge, immersion as well as of the interface itself. This questionnaire was based on a visual scale which the patient used regularly to report on fatigue. The answers recorded in this first questionnaire were then translated into items inspired by the game experience questionnaire scale (IJsselsteijn et al., 2013). This scale comprises five items (0: not at all, 1: slightly, 2: moderately, 3: fairly and 4: extremely) in French version. The patient enjoyed the game (3) and was immersed, as he lost perception of the time spent on it. Indeed, he reported playing only 8 to $10 \mathrm{~min}$ instead of the actual 30 minutes played. The game was adapted to the patient, i.e., not very easy (2) and not very difficult (3). 
Table 1 Subjects

\begin{tabular}{lllllllll}
\hline Patient & 1 & 2 & 3 & 4 & 5 & 6 & 7 \\
\hline Age & 52 & 67 & 71 & 58 & 72 & 38 & 73 \\
Gender & W & M & M & M & M & W & M \\
Affected arm & L & L & L & L & R & L & L \\
Dominant hand & R & R & R & R & R & R & R \\
Number of weeks after stroke & 11 & 11 & 23 & 30 & 7 & 16 & 12 \\
Hemineglect & No & L & L & L & No & L & L \\
Attention disorders & No & Yes & Yes & No & No & No & Yes \\
\hline
\end{tabular}

The tasks were easy to understand (2) and the game's objective was clear to him (3). Finally, the therapists reported that the game was interesting, and appeared well-adapted to stroke patients. They also recommended the game be included in a therapy program.

To evaluate the effects of the adaptation strategy on patients' training outcomes, we conducted an experiment with stroke patients. Next, we describe the experiment protocol.

\subsection{Participants}

Seven post-stroke patients aged 38 to 73 years (average age was 61.57; standard deviation was 12.06) were asked to complete game tasks using their impaired arm. One patient played bi-manually (i.e., with two arms) because of his severe disability. The group totaled 7 patients and comprised 5 men and 2 women. The average number of weeks after stroke was 15.71, with a standard deviation of 7.37. All patients were able to use the graphics tablet's mouse. Selecting from the five choices of the visual scale (0: not at all, 1: slightly, 2: moderately, 3: fairly and 4: extremely), the patients reported that the mouse was moderately difficult to use (2). Table 1 shows the patients' main characteristics. Our inclusion criteria were not restrictive. In fact, the only criterion was the participant's ability to use the game device (i.e., the mouse of the graphics tablet). We selected patients without severe cognitive deficits and who were able to play video games. They presented with mild language deficits as assessed with the Boston Naming Test (greater than 3/5) and mild Unilateral Neglect (less than 8 bells).

\subsection{Design and hypotheses}

We focused our study on the assessment of the difficulty strategy. Thus, three conditions of this independent variable were reviewed: DDA (Dynamic Difficulty Adaptation strategy), IDA (Incremental Difficulty Adaptation strategy) and Random (no difficulty strategy).

Dynamic difficulty adaptation (DDA): As explained above, DDA is based on the player's profile. It aims to dynamically compute appropriate challenges 
for patients by maximizing their efforts without exceeding their abilities. The objective is to control the patient's effort during a game session to prevent fatigue and therefore improve training outcomes.

Incremental difficulty adaptation (IDA): IDA has been proposed in many studies in the literature (Rabin et al. , 2011; Mihelj et al., 2012). This strategy consists in gradually providing the patient with the easiest to most difficult tasks. To do so, the distance required to reach the targets is progressively increased, thereby leading the patient to put more effort into the game session. The increase in difficulty is the same for all patients and is not based on the patient's individual performance.

No difficulty adaptation (Random): This control strategy consists in providing patients with targets in different locations of the workspace. The positions of the targets are randomly generated in the game level without taking patient performance or profile into account.

The objective of our study was to evaluate the effects of the difficulty strategy on the player's training outcomes, especially the total number of tasks attempted, the number of tasks successfully completed, and the cumulative distance covered. The general null hypothesis is: $H 0_{m}$ : the difficulty strategy does not influence the patient's performance during the therapeutic session. Thus, the following null hypotheses were defined:

- $H 0_{a}$ : the difficulty strategy has no effect on the number of tasks attempted by the player during a therapeutic session.

- $H 0_{b}$ : the difficulty strategy has no effect on the number of tasks successfully completed during a therapeutic session.

- $H 0_{c}$ : the difficulty strategy has no effect on the movement amplitude (distance) during a therapeutic session.

We also retained two questionnaire measures: perceived game duration and perceived difficulty. Thus, the following null hypotheses were stated:

- $H 0_{d}$ : there is no difference between the three games in terms of perceived difficulty.

- $H 0_{e}$ : there is no difference between the three games in terms of perceived game duration.

Finally, patients were interviewed to evaluate player experience.

We used a repeated-measure, single-blinded design. Each patient was asked to perform an assessment exercise and then play a randomly selected game version (see Fig. 1). At the beginning of the session, the patient was given instructions on how to play a PRehab level, and shown an example. Then, the sessions were randomized between patients. Each patient received 20 minutes of training (i.e., to play a game version). The training was given in all the sessions.

All participants used the same device, an Intuos A3 graphics tablet (see Fig. 9). The experiment was conducted over a two-week period. Each patient participated in three rehabilitation sessions (one per day, at the same time 


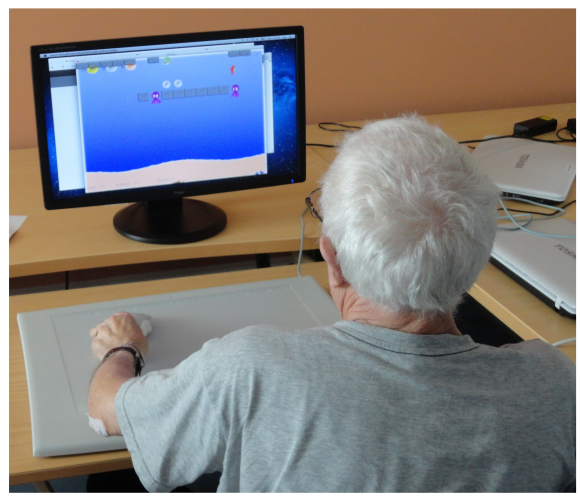

Fig. 9 A patient playing PRehab during the experiment.

every day). Two therapists participated in the rehabilitation sessions to observe patient-game interactions. At the end of each game session, the patients rated their experience using a 5-item visual scale (0: not at all, 1: slightly, 2: moderately, 3: fairly and 4: extremely).

Each game version generated game levels according to a difficulty strategy: Cat game (Random), Rabbit game (IDA) and Turtle game (DDA). The three game versions were similar in terms of mechanics and dynamics, but offered different graphics. In particular, the characters' image and animation features were different, as were the backgrounds. Game feedback, number and position of PRehab coins were the same in the three sessions. The reason we presented three different graphic environments was to help patients differentiate between the three game versions. In our previous clinical trials, we had observed that patients were unable to clearly determine whether the difficulty of the game was due to the device or to the software.

The number of targets that the patient reaches in the game depends on his or her speed and the importance of the efforts he or she makes. All three games generated real-time levels and targets. The difficulty strategy only affected the position of the targets, and did not have any impact on their appearance timing. The time required to reach a target and the time of appearance of the next target were similar across the three game versions. Once the patient reached all the targets of a game scene, the next scene appeared with new targets. When the patient failed to reach a target within the time allocated, the target disappeared and the task was considered unsuccessful. This time limit was set to 20 seconds in the three game versions. 
Table 2 ANOVA test results. $D F_{n}$ : degrees of freedom in the numerator; $D F_{d}$ : degrees of freedom in the denominator; $F$ ratio: statistic test for the ANOVA; $p$ : p-value, $\eta^{2}$ : partial eta-squared; HSD.05: Tukey's HSD test. The level of power was .80 .

\begin{tabular}{llllllc}
\hline Dependant variable & $D F_{n}$ & $D F_{d}$ & $F$ & $p<.05$ & $\eta^{2}$ & $H S D .05$ \\
\hline Number of tasks & 2 & 10 & 5.859 & 0.020 & 0.539 & 6.79 \\
Number of completed tasks & 2 & 10 & 7.705 & 0.009 & 0.606 & 6.92 \\
Distance covered & 2 & 10 & 4.395 & 0.042 & 0.467 & 27.48 \\
\hline & & & & & \\
\hline Dependant variable & DDA vs. Random & DDA vs. IDA & IDA vs. Random \\
\hline Number of tasks & & $p<0.17$ & & $p<1$ & & $p<0.14$ \\
Number of completed tasks & & $p<0.48$ & & $p<0.31$ & & $p<1$ \\
Distance covered & & $p<0.02$ & & $p<0.05$ & & $p<0.42$ \\
\hline
\end{tabular}

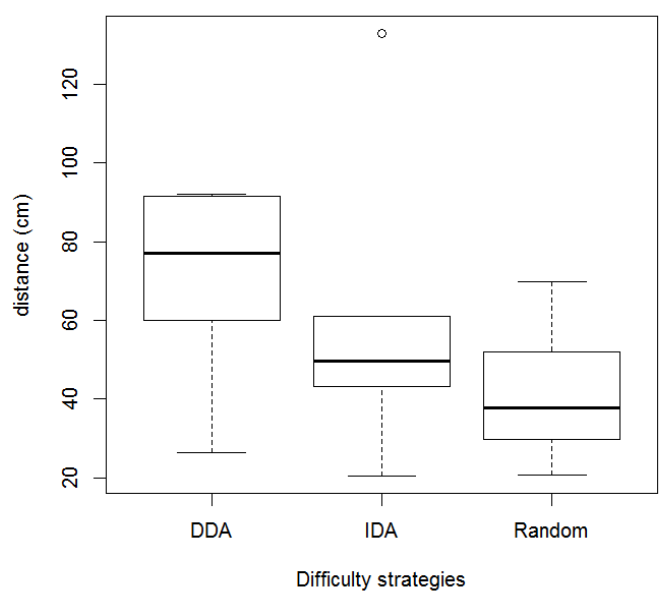

Fig. 10 Distance covered in the three difficulty strategies.

\section{Results}

In this section, we examine the results of our experiment. We first focus on the analysis of the patient performance measures, and then review the questionnaire measures. The analysis is based on the data for six patients only. Patient number 2 (see Table 1) stopped playing the game due to his health condition. The therapist who observed the session reported that this patient was tired and had complained about pains prior to the experiment. 


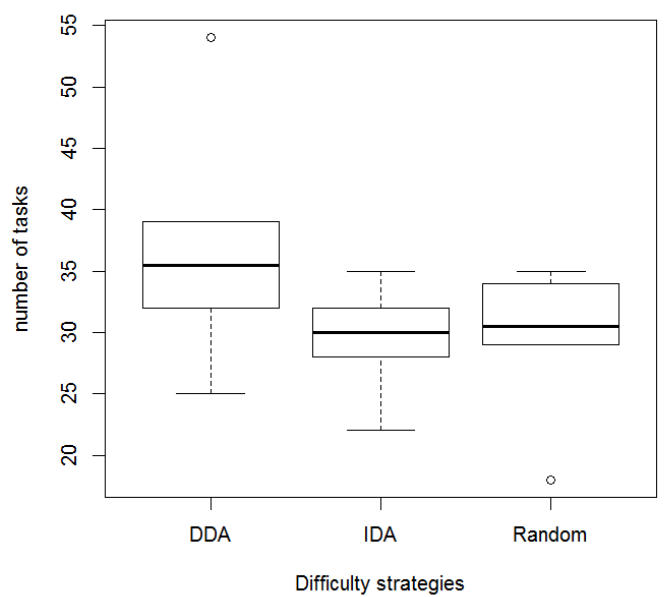

Fig. 11 Number of tasks in the three difficulty strategies.

\subsection{Performance measures}

All statistical analyses were performed using R (http://www.r-project.org), with a significance threshold of $p<0.05$. We first ran a multivariate analysis of variance (MANOVA) to investigate the mean differences on the dependent variables. Then, this analysis was complemented with a one-way ANOVA with repeated measures. The normality of the distribution was assessed with the Kolmogorov-Smirnov test. Finally, post-hoc pairwise comparisons were made using the Tukey HSD and Bonferroni tests to identify specific differences and to adjust errors.

The MANOVA test rejected the null hypothesis $H 0_{m}$. It revealed a significant effect of the difficulty strategy on patient performance (Wilks' Lambda= $0.10 ; \mathrm{F}=2.38 ; p<0.02)$.

The ANOVA conducted on the three dependent variables indicated that the difficulty strategy significantly influenced the total distance, but not the number of tasks or the number of successes (see Table 2). The DDA strategy increased the distance covered, compared to the control strategies (see Fig. 10). Based on Cohen et al. (1988) measures, effect size was medium for the three ANOVAs $(f<0.059)$. The mean and variance for the three strategies are shown in Table 3 .

\subsection{Questionnaire measures}

The perceived difficulty of the game tasks was evaluated with a 5 -item visual scale (0: not at all, 1: slightly, 2: moderately, 3: fairly and 4: extremely). The 
Table 3 Mean and standard deviation

\begin{tabular}{llll}
\hline Dependant variable & Random & IDA & DDA \\
\hline Number of tasks & $29.5 \pm 6.1$ & $29.5 \pm 4.3$ & $36.8 \pm 9.7$ \\
Number of completed tasks & $28 \pm 7.2$ & $26.6 \pm 8.7$ & $35.8 \pm 11.1$ \\
Distance covered $(\mathrm{cm})$ & $41.3 \pm 17.4$ & $59.5 \pm 38.4$ & $70.7 \pm 25.6$ \\
\hline
\end{tabular}

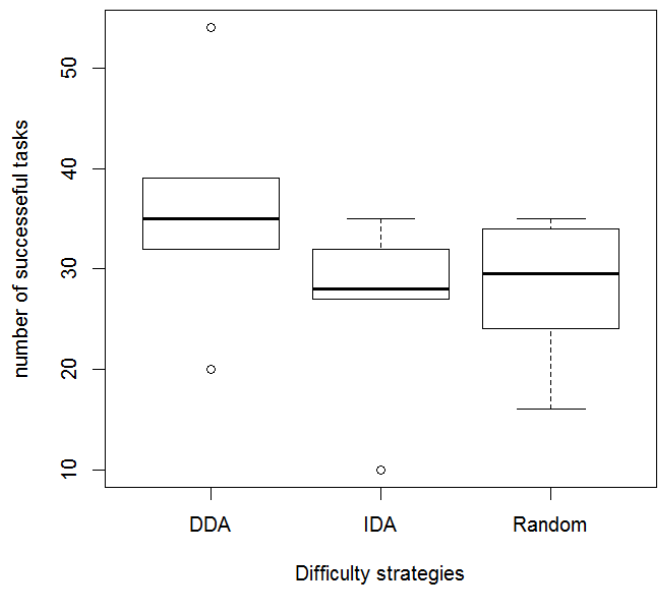

Fig. 12 Number of successful tasks in the three difficulty strategies.

median values showed that the DDA-based game was moderately difficult (2). The Friedman test did not reject the null hypothesis $H 0_{d}$ (Friedman chisquared $=2.8 ; d f=2 ; p<0.24)$.

We also evaluated patient-perceived game duration at the end of each game session. The ANOVA test did not reject the null hypothesis $H 0_{e}(\mathrm{~F}(2,10)=3.5$, $p=0.06)$. However, five patients reported that each session lasted about 3 to 15 minutes, when actual sessions time was 20 minutes.

\section{Discussion}

The online adaptation of game difficulty has become the focus of many studies on rehabilitation games. However, most of the rehabilitation games reviewed in the literature are based on adhoc adaptation solutions. These adaptation techniques depend on game metrics and lack reusability. They usually rely on two strategies: (i) dynamic difficulty adaptation aims to increase and/or decrease the difficulty during the game session based on the patient's performance; (ii) incremental difficulty adaptation seeks to progressively increase the difficulty during the game session. To the authors' knowledge, no study to date has investigated the evaluation of the effect of these difficulty strategies 
on patient performance. In (Cameirao et al., 2010), the study was limited to the evaluation of the acceptability of the system and the validation of the correspondence of the game measures with the difficulty. In BrightArm (Rabin et al. , 2011), a study based on an incremental difficulty strategy, 5 participants increased their active shoulder range by 15 to 25 flexion degrees and by 10 to 30 abduction degrees. However, though this study did assess the impact of the game on shoulder flexion, it failed to evaluate the adaptation strategy itself.

In this paper, we first described a generic adaptation technique based on a Monte Carlo tree search algorithm. This technique is used in serious games designed for upper-arm rehabilitation based on pointing tasks. Second, we described PRehab, a new rehabilitation game designed to dynamically generate game levels. Finally, we studied the effect of the proposed DDA strategy on the training outcomes and compared it to two control strategies.

The results of the experiment show that DDA improved patient performance in terms of movement amplitude (distance). Increasing movement amplitude is a key objective in rehabilitation for stroke patients. Since DDA is based on controlling the patients' effort, it prevents their fatigue. At the start of the therapy session, DDA provides challenging, though achievable, tasks to the patients. Then, if the patient successfully completes the tasks, DDA maximizes this effort, by increasing the difficulty of the subsequent tasks. If the patient fails to complete several successive tasks, the algorithm progressively decreases the difficulty to prevent fatigue. The adaptation of the difficulty ensures that the tasks remain challenging without requiring a disproportionate level of effort. This is achieved through the concept of the ability zone, which models the patient's abilities during the game session.

Although the three game versions share the same mechanics and success/failure conditions (e.g., the number of tasks, the time required for the appearance of targets), we observed that the patients completed more tasks in the Turtle game (based on DDA). However, despite differences (number of tasks attempted and of tasks successfully completed) between the three difficulty strategies (see Fig. 11 and Fig. 12), the statistical tests were not significant. This may be explained by the short duration of the game session (20 minutes) in our experiment. Specifically, we observed that the number of unsuccessful tasks at the end of the session (in the 5 last minutes) was higher (80\%) in the IDA and Random strategies than in the DDA strategy (50\%). This may be explained by patient fatigue in IDA and Random. Future experiments should attempt to verify this assumption by studying the long-term effect of difficulty strategies.

We also examined the effects of the difficulty strategy on the patients' immersion and motivation. To evaluate immersion, we recorded the patients' perceived game duration for each game session. In general, as a result of immersion, people tend to lose the sense of time passing (Sanders et al., 2010). Similarly, serious games may affect immersion by reducing the perception of time passing. As a result, patients could spend more time on a game and thusly put more efforts into the therapeutic session. Many factors may influence the player's immersion. Our study showed that the difficulty strategy did not in- 
fluence perceived game duration. Nevertheless, most patients reported that sessions lasted between 3 and 15 minutes, while actual session duration was 20 minutes for all the games. Thus, we believe that there are other elements in serious games that may influence the patients' immersion. In future works, we plan to study game elements that influence the immersion (e.g. graphics, feedback, story). We will also compare the effects of serious games on patient immersion with those of traditional physical rehabilitation programs.

Furthermore, we assessed patient-perceived difficulty of game tasks. We believe that when patients perceive that tasks are not very difficult and thusly successfully meet game objectives, they become motivated. This assumption is based on Kukla (1972) analysis of achievement behavior in terms of costbenefit judgment. According to this analysis, as long as the subject perceives that the effort is worthwhile, the intended effort is likely to be inversely proportional to the perceived probability of success (Kukla, 1972; Brehm et al., 1989). The difference between the three strategies in terms of perceived difficulty was not significant. However, a stroke may impair the ability of some patients to make sound judgments about the difficulty of an activity. Therefore, the difficulty strategy may influence perceived difficulty in stroke patients. Future experiments should attempt to confirm this assumption with a classification scheme including only patients with mild or very mild cognitive deficits.

When evaluating PRehab, most patients found the objectives of the game clear and the interface adequate. A patient said: "I like the game, it is like Mario !", and It is easy and fun to play". Another patient who also enjoyed the game said: "I told other patients about the game!"

In the course of the interview, we asked patients which game they thought was the most adaptive. All of them answered that they preferred the Turtle game (DDA). Many patients mentioned that they had difficulties reaching targets in the Rabbit (IDA) and Cat (Random) game versions. For example, concerning the Rabbit game version, a patient reported that: "The game was very difficult at the end and the targets were on the left side". Another patient felt that the Turtle game was the most adaptive one: "I understand: the game took my evaluation into account to adapt itself to my abilities!"

In these interviews, all the patients reported that they would likely enjoy using PRehab in their rehabilitation programs. Several patients even insisted on continuing to play despite their health condition and pain. For example, during a game session, a patient said: "I can stand the pain, I want to continue to play". We therefore believe that adaptive serious games are promising rehabilitation tools in that they foster and sustain patient motivation.

\section{Limitations and future work}

The proposed adaptation technique seeks to improve training outcomes. It is based on a player profiling technique that centers on the short-term evaluation of the patient's abilities. By taking into account the daily health condition of stroke patients, it allows the game to dynamically adapt the task to the 
patient's abilities. Based on our results, this technique looks very promising. However, the recovery of stroke patients depends on various factors and a longterm evaluation of their abilities may be useful to develop training strategies throughout the therapy process. Future works should integrate both shortand long-term evaluations to enhance the adaptation strategy.

Other limitations to the study have to be mentioned. First, the number of participants was limited. In addition, most subjects suffered from left hemiplegia and cognitive deficits. This may have influenced the number of tasks completed. Due to attention deficits, four patients exhibited slower reaction times.

Second, to help patients distinguish between the three difficulty strategies, we designed a game with three different graphic environments. We do not believe that this affected the results of the study. The patients reported that they enjoyed all versions. They viewed these versions as different difficulty levels of the same game. We therefore assumed that this bias did not influence the study outcomes. In future work, however, we plan to study the effect of the game's characters and feedback on patient performance and motivation.

Finally, the game tasks were generated at the same rate in all different strategies. In future work, this information will be used by DDA to control the "training interval" for a patient. We will also consider the time constraints on tasks and the cognitive challenges for patients at an advanced recovery stage. Furthermore, in our experiment, the adaptation technique always started in difficult mode and was adjusted throughout the game session. In future work, we will attempt to vary the starting mode in order to analyze its influence on training outcomes. Pre- and post-evaluations of patient performance and ability will be considered for short- and long-term evaluations.

\section{Conclusion}

In this paper, we presented a dynamic difficulty adaptation technique for rehabilitation games based on pointing tasks (i.e., reaching targets). The technique relies on the player's profile by integrating a short-term prediction of the player's abilities. The objective is to improve the patients' training outcomes during a therapeutic session. This adaptation technique was used to adapt PRehab, a single-player game based on pointing tasks.

Our experiment sought to study the effects of game difficulty on the performance of stroke patients. The proposed difficulty adaptation strategy was compared to two other control strategies: a random strategy and an incremental difficulty strategy. The results of the experiment show that the dynamic difficulty adaptation increased patient performance in terms of movement amplitude (distance), an important parameter in stroke rehabilitation.

We also discussed in this paper the importance of the design of rehabilitation games. We presented the design of PRehab, a serious game based on pointing tasks. Designing PRehab allowed us to evaluate the difficulty strategy without influencing the game's mechanics and rules. Thus, the game may 
be used in future research studies with new difficulty strategies. PRehab also makes it easy for therapists to modulate the difficulty strategy.

Our priorities for future work are to enhance the adaptation strategy by varying time constraints on tasks and adding cognitive challenges for stroke patients in advanced recovery stages. We also plan to study the impact of graphic elements (game characters and feedback) on patient performance and motivation. Pre- and post-evaluations will be included for short- and longterm tests. Finally, in order to further evidence the benefits of serious games in the context of stroke rehabilitation, we plan to study the differences between traditional physical therapy and PRehab-based therapy.

\section{References}

M. Annett, F. Anderson, D. Goertzen, J. Halton, Q. Ranson, W. Bischof, P. Boulanger, Using a multi-touch tabletop for upper extremity motor rehabilitation, Proceedings of the 21st Annual Conference of the Australian Computer-Human Interaction Special Interest Group, Melbourne, Australia, pp. 261-264 (2009)

S. Avila-Sansores, F. Orihuela-Espina, L. Enrique-Sucar, Patient Tailored Virtual Rehabilitation, Converging Clinical and Engineering Research on Neurorehabilitation, Springer, pp. 879-883 (2013)

M. Beynon, W. Beynon, Mediating intelligence through observation, dependency and agency in making construals of malaria, Intelligent Tutoring Systems Conference, Chania, Crete, Greece, pp. 664-665 (2012)

J. Burke, M. McNeill, D. Charles, P. Morrow, J. Crosbie, S. McDonough, Serious games for upper limb rehabilitation following stroke, Conference in Games and Virtual Worlds for Serious Applications, Coventry, United Kingdom, pp. 103-110 (2009)

J. Burke, M. McNeill, D. Charles, P. Morrow, J. Crosbie, S. McDonough, Optimising engagement for stroke rehabilitation using serious games, Journal of the Visual Computer, Springer, 25, pp.1085-1099 (2009)

J. Brehm, E. Self, A The intensity of motivation, Annual review of psychology, 40, pp.109-131(1989)

M.S. Cameirão, S.B. Badia, E.D. Oller, Neurorehabilitation using the virtual reality based Rehabilitation Gaming System: methodology, design, psychometrics, usability and validation, Journal of neuroengineering and rehabilitation, 7, 48 (2010)

L. Chittaro, R. Ranon, E. Carchietti, A. Zampa, E. Biasutti, L. De Marco, A. Senerchia, A knowledge-based system to support emergency medical services for disabled patients, Journal of Artificial Intelligence in Medicine, Elsevier, pp. 176-180 (2009)

M. C. Cirstea, M.F. Levin, Improvement of arm movement patterns and endpoint control depends on type of feedback during practice in stroke survivors, Journal of Neurorehabilitation and Neural Repair, 21, pp. 398-411 (2007) 
J. Cohen J. Statistical Power Analysis for the Behavioral Sciences. New York, NY: Routledge Academic, pp. 23-29 (1988)

C. Conati, H. Maclaren, Modeling user affect from causes and effects, User Modeling and User-Adapted Interaction: The Journal of Personalization Research, pp. 4-15 (2009)

C. Conati and M. Manske, Evaluating Adaptive Feedback in an Educational Computer Game, 9th international conference on intelligent virtual agents, Amsterdam, pp.146-158 (2009)

M. Czikszentmihalyi, Flow: The psychology of optimal experience, Praha: Lidové Noviny (1991)

D. Deligneieres, La perception de la difficulte dans les taches motrices, HDR report, university of Montpellier 1, pp. 23-29 (2010)

B. H. Dobkin, Rehabilitation after stroke, New England Journal of Medicine, 352, pp. 1677-1684 (2005)

A. Gouaïch, N. Hocine, L. Van Dokkum, D. Mottet, Proceedings of the 2nd ACM SIGHIT International Health Informatics Symposium, Miami, USA, pp. 5-12 (2012)

D. Goude, S. Björk, M. Rydmark, Game design in virtual reality systems for stroke rehabilitation, Journal of studies in health technology and informatics, pp. 146-148 (2007)

N. Hocine, A. Gouaich, Therapeutic Games Difficulty Adaptation: An Approach Based on Player Ability and Motivation, Proceddings of 16th International Conference CGAME 2011, USA, pp. 257-261 (2011)

J. Howcroft, S. Klejman, D. Fehlings, V. Wright, K. Zabjek, J. Andrysek, E. Biddiss, Active video game play in children with cerebral palsy: Potential for physical activity promotion and rehabilitation therapies, Archives of physical medicine and rehabilitation, pp. 1448-1456 (2012)

R. Hunicke, V. Chapman, AI for dynamic difficulty adjustment in games, Challenges in Game Artificial Intelligence AAAI Workshop, pp. 91-96 (2004)

W.A IJsselsteijn, Y. De Kort, K. Poels, The Game Experience Questionnaire: Development of a self-report measure to assess the psychological impact of digital games. Manuscript (2013)

M. Khademi, H. Mousavi, C.V. Lopes, L. Dodakian, S.C. Cramer, Haptic Augmented Reality to monitor human arm's stiffness in rehabilitation, IEEE EMBS Conference on Biomedical Engineering and Sciences, Malaysia, pp. 892-895 (2012)

J. Kivikangas, I. Ekman, G. Chanel, S. Järvelä, M. Salminen, B. Cowley, P. Henttonen, N. Ravaja, Review on psychophysiological methods in game research, Journal of Gaming and Virtual Worlds, 3, pp. 181-199 (2010)

L. Kocsis, C. Szepesvari, Bandit based monte-carlo planning, Machine Learning: ECML 2006, 282-293 (2006)

A. Kukla, Foundations of an attributional theory of performance, Psychological Review (1972)

G. Kwakkel, R.C. Wagenaar, T.W. Koelman, G.J. Lankhorst, J.C. Koetsier, Effects of intensity of rehabilitation after stroke a research synthesis, Journal of Stroke, 28 (1997) 
Left 4 Dead video game, www.14d.com, 2008

F. Levin, H. Sveistrup, S. Subramanian, Feedback and virtual environments for motor learning and rehabilitation, Schedae, 1, pp. 19-36 (2010)

B. Magerko, B. Stensrud, L. Holt, Bringing the schoolhouse inside the box-A tool for engaging, individualized training, pp. 23-29 (2006)

T. Markow, Mobile music touch: using haptic stimulation for passive rehabilitation and learning, Doctoral dissertation, Georgia Institute of Technology (2012)

Max Payne video game, www.maxpayne.com, 2001

J. McCuaig, R. Gauthier, Interoperability for ITS: an ontology of learning style models, Intelligent Tutoring Systems, Crete, pp. 671-672 (2012)

M. Mihelj, D. Novak, M. Milavec, J. Ziherl, A. Olenšek, M. Munih, Virtual rehabilitation environment using principles of intrinsic motivation and game design, Presence: Teleoperators and Virtual Environments, 21, pp. 1-15 (2012)

T. Murray, I. Arroyo, Toward measuring and maintaining the zone of proximal development in adaptive instructional systems, Intelligent Tutoring Systems, France, pp. 749-758 (2002)

S. Natkin, C. Yan, S. Jumpertz, B. Market, Difficulty scaling of game AI, International Conference on Digital Games Research Association, Tokyo, Japan, pp. 33-37 (2007)

T. Nef, R. Riener, Three-Dimensional Multi-Degree-of-Freedom Arm Therapy Robot (ARMin), Neurorehabilitation Technology, Springer, pp. 141-157 (2012)

PCG, Wiki page for procedural content generation site : http://pcg.wikidot.com (2014)

N. Peirce, O. Conlan, V. Wade, Adaptive educational games: Providing noninvasive personalised learning experiences, Second IEEE International Conference on Digital Games and Intelligent Toys Based Education, Canada, pp. 28-35 (2008)

M. Pirovano, R. Mainetti, G. Baud-Bovy, P.L. Lanzi, N. Borghese, Selfadaptive games for rehabilitation at home, IEEE Conference on Computational Intelligence and Games (CIG), Granada, pp. 179-186 (2012)

PRehab, serious game for stroke rehabilitation, university of Montpellier, video of the game : http://www.youtube.com/watch?v=cfTv9Dtc5Ww

L. Pugnetti, L. Mendozzi, E.A. Attree, E. Barbieri, B. Brooks, C. Cazzullo, A. Motta, F.D. Rose, C. Psychol, Probing memory and executive functions with virtual reality: Past and present studies, CyberPsychology \& Behavior, 1, pp. 151-161 (1998)

B. Rabin, G. Burdea, J. Hundal, D. Roll, F. Damiani, Integrative motor, emotive and cognitive therapy for elderly patients chronic post-stroke A feasibility study of the BrightArm rehabilitation system, International Conference on Virtual Rehabilitation, Switzerland, pp. 1-8 (2011)

P. Rani, N. Sarkar, L. Nilanjan, C. Liu, Maintaining optimal challenge in computer games through real-time physiological feedback, Proceedings of the 11th International Conference on Human Computer Interaction, Las 
Vegas, USA, pp. 184-192 (2005)

R. Rodrigo, R. Fernández-Gajardo, R. Gutiérrez, J.M. Matamala, R. Carrasco, A. Miranda-Merchak, W. Feuerhake, Oxidative Stress and Pathophysiology of Ischemic Stroke: Novel Therapeutic Opportunities, Journal of Oxidative Stress and Pathophysiology of Ischemic Stroke: Novel Therapeutic Opportunities, CNS \& neurological disorders drug targets, pp. 23-29 (2013)

D. Rojas, B. Kapralos, S. Cristancho, K. Collins, A. Hogue, C. Conati, A. Dubrowski, Developing effective serious games: The effect of background sound on visual fidelity perception with varying texture resolution, Journal of Studies in health technology and informatics, 173, pp. 386-392 (2012)

T. Sanders, P. Cairns, Paul, Time perception, immersion and music in videogames, Proceedings of the 24th BCS Interaction Specialist Group Conference, Swinton, UK, pp. 160-167 (2010)

K. Sherlock, Plays, Acts and Scenes in Structure, Drama Writing, Grossmont College (2005)

P. Spronck, M. Ponsen, I. Sprinkhuizen-Kuyper, E. Postma, Adaptive game AI with dynamic scripting, journal of Machine Learning, 63, pp. 23-29 (2006)

P. Spronck, I. Sprinkhuizen-Kuyper and E. Postma, Difficulty Scaling of Game AI, Proceedings of the 5th International Conference on Intelligent Games and Simulation, Belgium, pp. 33-37 (2004)

S. Tavener, D. Perez, S. Samothrakis, S. Colton, A Survey of Monte Carlo Tree Search Methods, IEEE Transactions on Computational Intelligence and AI in Games, pp.1-43 (2012)

T. Tijs, D. Brokken, W. IJsselsteijn, Dynamic game balancing by recognizing affect, Fun and Games, Springer Berlin Heidelberg, pp. 88-93 (2008)

J. Togelius, G. Yannakakis, K. Stanley, C. Browne, Search-based procedural content generation Applications of Evolutionary Computation, IEEE Transactions on Computational Intelligence and AI in Games, 3, pp. 141-150 (2010)

B. Varkuti, C. Guan, Y. Pan, K. Phua, K. Ang, C. Kuah, K. Chua, B. Ang, N. Birbaumer, R. Sitaram, Resting state changes in functional connectivity correlate with movement recovery for BCI and robot-assisted upper-extremity training after stroke, journal of Neurorehabilitation and Neural Repair, 27, pp. 53-62 (2013)

O. R. Vincent, O. Folorunso, A descriptive algorithm for sobel image edge detection, Proceedings of Informing Science and IT Education Conference (InSITE), USA, pp. 97-107 (2009)

J. Wertsch, The zone of proximal development: Some conceptual issues, New Directions for Child and Adolescent Development, pp. 7-18 (1984)

World Health Organization (WHO): The top 10 causes of death (2013)

G. N. Yannakakis, J. Hallam, Real-time Adaptation of Augmented-Reality Games for Optimizing Player Satisfaction, IEEE Symposium of Computational Intelligence and Games, Australia, pp. 103-110 (2008)

A. Zook, M. Riedl, A Temporal Data-Driven Player Model for Dynamic Difficulty Adjustment, The Eighth Annual AAAI Conference on Artificial Intelligence and Interactive Digital Entertainment, USA, pp. 23-29 (2012) 


\section{Authors vitae}

1) N. Hocine: University of Montpellier, Laboratory of Computer Science, Robotics, and Microelectronics (LIRMM), CNRS, 161 r. Ada, 34095, Montpellier, France

Dr. N. Hocine obtained her Ph.D of informatics at the University of Montpellier in 2013. She worked as temporary Assistant Professor at the University of Montpellier and then at the University of Cote d'Opale in Dunkerque, France. Her research work deal with software solutions for the adaptation of user-centered systems and its applications for health. Hocine's thesis has been introduced in the context of Heath Oriented Game Engine Project which involved LIRMM laboratory, industrial partners and physicians. The thesis was founded on serious games adaptation in post-stroke functional rehabilitation. Hocine's current research objectives concern user modeling and adaptation techniques in health informatics.

2) A. Gouaich: University of Montpellier, Laboratory of Computer Science, Robotics, and Microelectronics (LIRMM), CNRS, 161 r. Ada, 34095, Montpellier, France

Dr. A. Gouaich is an Associate Professor at the University of Montpellier. He received his Ph.D. of Computer Science in 2005 at the University of Montpellier within the area of multi-agent systems and ubiquitous computing. His work concerned the definition of concepts, models and middlewares for ubiquitous and mobile services using software agents. Dr. Gouaich joined the agent group at Motorola Labs (Paris). In 2006, he worked as a research fellow in PolicyGrid project at the University of Aberdeen (Scotland). In 2007, Dr. Gouaich joined, as an assistant professor, LIRMM laboratory. His current research activities concern adaptive games and serious games using agent technologies.

3) S. A. Cerri: University of Montpellier, Laboratory of Computer Science, Robotics, and Microelectronics (LIRMM), CNRS, 161 r. Ada, 34095, Montpellier, France

S.A. Cerri is Professor of informatics at the University of Montpellier. He graduated in Physics in Pisa (1971); worked as tenured staff member at the Universities of Pisa, Amsterdam, Milan and Montpellier; as visiting professor at Brussels and Nice-Sophia Antipolis. His main scientific contributions, since his thesis about learning classical mechanics by simulation, concern the intersection between Informatics and Human Learning, combining Computation, Cognition and Communication. He authored about 130 papers in international journals, peer reviewed conference proceedings, chapters of books; edited 8 books; was invited speaker at conferences, Universities and Industries (45); participated to more than 25 European R/D Projects. Between 2005 and 2010 he was Deputy Director of the Montpellier Laboratory of Informatics, Robotics, and Microelectronics that is a French cross-faculty research entity of the University of Montpellier and the National Centre for Scientific Research (CNRS). Stefano Cerri developed a unified formal model for human and artificial Agents mutually delivering stateful services. The main challenge 
being the personalization of dialogues, his main concern derived from his early research on student modeling, having as a consequence the goal of personalization in games. Currently, his main commitment is about Web Science and related studies concerning models, infrastructures and applications supporting connected communities of human Agents jointly working, playing, learning and contributing to politics.

4) D. Mottet: University of Montpellier, EuroMov, 700 av Pic St Loup, 34090 Montpellier, France

Dr. D. Mottet is Professor of Movement Sciences at the University of Montpellier, in charge of the Technological Platform of the EuroMov Center. He received a Master in Computer Sciences, a MD degree and a Ph.D. in Human Movement Sciences from Aix-Marseilles University. Dr Mottet uses control theory and dynamical systems theory to shed light on data from behavioral experiments on humans pushed at their limits (speed, accuracy, fatigue). Most of his research time is now devoted to ICT for health, especially to improve rehabilitation of the upper-limb after a stroke

5) J. Froger: Movement to Health laboratory (M2H), University Hospital of Nimes and Montpellier, France

Dr. J. Froger is a physician in physical medicine and rehabilitation. He received his BA of physical medecine and rehabilitation from the university of Lille in 1999. He is currently responsible of the neurological rehabilitation unit of the Hospital and CHU of Nimes. His research concerns the study of disorders observed in stroke patients to improve their rehabilitation. Dr. J. Froger currently focuses on the study of the assessment of new rehabilitation programs for the upper and lower arm based on robotics, virtual reality and serious games.

6) I. Laffont Movement to Health laboratory (M2H), University of Montpellier, University Hospital of Nimes and Montpellier, France

Prof. I. Laffont leads the Physical Medicine and Rehabilitation Department of Montpellier Hospital in France. She received her Ph.D degree in Movement Sciences in 2005. The main topic of her research concerns Neuro-rehabilitation and Neuro-plasticity in upper limb movements of stroke patients. She currently works with three-dimensional motion analysis (Polhemus*, Zebris*) aiming to understand neurological mechanisms underlying movement restoration, adaptation or compensation after lesion. The purpose of her works aims to develop and assess innovative rehabilitation programs after stroke, including development of adaptive serious games, robotic aids, multi-modal feedbacks, and functional electric stimulation. 